\title{
Modelos predictivos de riesgo cardiovascular
}

Eduardo Antonio Burgos Martínez ${ }^{1}$, Andrés Felipe Ramírez², Erika Sofía Villamil²

Médico Internista y Cardiólogo. Coordinador del servicio de cardiología de la clínica IMAT Oncomédica.

2 Estudiante de décimo semestre, Facultad de Medicina, Fundación Universitaria Juan N, Corpas.
Correspondencia: Dr. Eduardo Antonio Burgos Martínez,edbuma2003@yahoo.es

Como citar: Burgos EA, RamírezAF, Villamil ES. Modelos predictivos de riesgo cardiovascular. Cuarzo 2016; 22 (2): 80-91.

Recibido: 18 de agosto de 2016.

Aceptado: 20 de octubre de 2016.

Publicado: 30 de diciembre de 2016.

Licencia creative commons (c) (i)

\section{Resumen}

Los modelos predictores estiman la probabilidad de desarrollar un evento cardiovascular (ECV) a futuro, son desarrollados con base en factores de poblaciones específicas y han sido validados internamente midiendo su discriminación y calibración, con el objeto de apoyar una intervención primaria y secundaria, no obstante, si se desea aplicarlos en una población distinta, se debe realizar una validación externa.

Diversas controversias rondan entorno a estos modelos, especialmente acerca de cuál aplicar en pacientes con diabetes y cuál usar en población colombiana. Se han planteado posturas sobre considerar la diabetes como equivalente de riesgo cardiovascular, evaluar estos pacientes igual que personas que carecen de ésta o brindar un enfoque separado; y aunque hay estudios que defienden diferentes posturas, es un tema que aún es controvertido y entre las guías internacionales aún no hay consenso sobre un modelo específico.

Por otra parte, se han llevado a cabo dos estudios en población colombiana usando la misma cohorte histórica, uno donde compara Framingham y PROCAM y otro SCORE, ACC/AHA y Framingham ajustado; el primer estudio determinó que PROCAM tenía mejor calibración y discriminación mientras que Framingham sobreestima el riesgo, y el segundo concluyó que ACC/AHA es mejor que SCORE aunque subestima el riesgo y en realidad ninguno lo estima correctamente. Pese a ello, las guías recomiendan el uso del Framingham ajustado.

Lo anterior refleja la necesidad de ahondar en la investigación sobre la estimación del riesgo cardiovascular especialmente en nuestro medio para brindar un enfoque acertado a la población colombiana, disminuyendo los ECV, mejorando la calidad de vida del paciente y dando una reducción en los costos al sistema de salud.

PALABRAS CLAVE: modelo cardiovascular, riesgo cardiovascular, puntaje de riesgo, modelos pronósticos 


\section{Predictive models of cardiovascular risk}

Predictive models estimate the likelihood of developing a cardiovascular event (CVD), are developed based on specific population factors and have been internally validated by measuring their discrimination and calibration, in order to support primary and secondary intervention, nonetheless, if it is necessary to make them in a different population, an external validation must be performed.

Several controversies surround these models, on the use of diabetes and the use in the Colombian population. Postures have been considered about considering diabetes as a cardiovascular risk equivalent, assessing these patients as well as providing a separate approach; and there are even studies that defend different positions, is an issue that is still controversial and among the international guides.

On the other hand, studies have been carried out in the Colombian population using the same historical cohort, one comparing Framingham and PROCAM and another SCORE, ACC / AHA and adjusted Framingham; The first study determined that there was better calibration and discrimination while Framingham overestimated the risk, and the latter concluded that ACC / AHA is better than SCORE, but estimates the risk and in fact not one correctly estimates it. In spite of this, the guides favor the use of the adjusted Framingham.

This reflects the need to delve into research on cardiovascular risk, especially in our environment, to provide a successful approach to the Colombian population, reducing CVD, improving the quality of life of the patient and reducing the costs of the health system.

KEYWORDS: cardiovascular model, cardiovascular risk, risk scoring, prognostic models.

\section{Introducción}

Los modelos de riesgo son ecuaciones que, mediante la evaluación de diversos predictores, permiten encontrar la probabilidad absoluta de que un resultado o evento ocurra (modelos pronósticos) en un tiempo determinado. En riesgo cardiovascular estos modelos pronósticos pueden ser usados tanto para prevención primaria como secundaria, ya que buscan identificar la probabilidad de que un evento cardiovascular de origen aterosclerótico se produzca, así la Organización Mundial de la Salud (OMS) promueve su uso en especial para prevención primaria mediante la puesta en marcha de dos estrategias, a saber: la primera está dirigida a la población general y se basa en la creación de políticas de salud pública encaminadas a cambiar los factores de riesgo cardiovasculares existentes; la segunda, busca combatir a nivel individual los diversos factores modificables que coexisten en el paciente mediante su identificación y adecuado manejo preventivo por parte del personal de salud durante la consulta (1-3).

De esta manera, los modelos se convirtieron en herramientas preventivas que evitan dejar solo al juicio clínico el acercamiento terapéutico permitiendo la evaluación objetiva del paciente en su contexto clínico, incluyendo tanto la carga como la severidad de la enfermedad, permitiendo con ello una mejor identificación en cuanto a seguridad y características costo-beneficio el óptimo tratamiento, aquel que permite disminuir en mejor medida la probabilidad de ocurrencia de eventos cardiovasculares con menores efectos adversos. Sumado a lo anterior los modelos mejoran la comunicación entre médico-paciente pues permiten clarificar expectativas, cambios en el estilo de vida y recomendaciones generales. Cabe mencionar como la creación de estas herramientas ha posibilitado la definición de los factores de riesgo cardiovascular tradicionales actuales, así como la identificación y evaluación tanto de nuevos marcadores como de posibles objetivos terapéuticos (4-6).

Actualmente, existen diversas controversias en torno al uso de estos modelos debido a la enorme diversidad de los mismos cada uno con diferencias en los predictores utilizados, resultados o eventos analizados, poblaciones usadas en el estudio de desarrollo y características propias de los diseños de este como aleatorización o seguimiento prospectivo o retrospectivo de los datos $(4,7)$. En 
pacientes diabéticos estas diferencias de opinión se hacen especiales debido a la consideración de esta enfermedad como equivalente de riesgo cardiovascular/infarto agudo al miocardio debatido ampliamente en la actualidad impactando drásticamente en el manejo especialmente farmacológico dado a este grupo de pacientes $(4,8-10)$.

Adicional a esto, los modelos en su mayoría tienen como base de estudio y extracción de datos población norteamericana y europea, se ha visto como la aplicación de un modelo en una población, diferente de la cual fue desarrollado, puede sobreestimar o subestimar el riesgo, caso en el cual el manejo instaurado pudiera ser el equivocado evitando cumplir con el objetivo principal de la aplicación de estos modelos en la práctica clínica. Considerando lo anterior, existen diversas herramientas epidemiológicas que pueden ser usadas para su validación externa y se hace necesario la aplicación de estas en los modelos que se deseen usar en poblaciones diferentes a las originales del estudio de desarrollo de los mismos $(4,6)$. En Colombia solo existe un estudio de validación externa para las ecuaciones de Framingham y PROCAM en población no necesariamente representativa de la existente en el país (11), a partir de este, la guía para la detección de riesgo cardiovascular del ministerio de salud (12), utiliza sus datos como base para la aplicación de Framingham en Colombia dejando entrever la necesidad de nuevas investigaciones de validación de las escalas predictivas de riesgo usadas en el ambiente clínico colombiano.

Así, en el presente documento, se busca dar una aproximación a las generalidades de los modelos de predicción de riesgo cardiovascular e indagar en y evidenciar la controversia existente en cuanto a la diversidad tanto de modelos actuales como de predictores empleados en los mismos, el uso de estos en pacientes diabéticos y la aplicación de modelos en poblaciones diferentes a las que fueron diseñados haciendo especial énfasis en los usados en el entorno de salud colombiana.

\section{Generalidades: Construcción de un modelo}

La generación de una escala de riesgo cardiovascular pasa por diversos puntos necesarios para obtener óptimos resultados al momento de su aplicación. El modelo en primera instancia debe desarrollarse, ser validado internamente, ser puesto a prueba en la práctica clínica, ajustarse o actualizarse; y en caso de ser usado en población diferente de la cual se originó, ser validado externamente y evaluar su impacto una vez implementado $(1,7)$. En cuanto al desarrollo, el primer paso es el planteamiento de diversas preguntas con el objetivo de buscar sus respuestas a partir de los datos obtenidos en los estudios Algunos de los interrogantes a responder son:

- ¿Qué enfermedad, resultado _o evento se busca predecir? Actualmente se busca predecir más de una enfermedad, los modelos de riesgo cardiovascular se han enfocado principalmente en la búsqueda de eventos derivados de enfermedad aterosclerótica, ejemplo de ello es el desarrollado por la asociación americana del corazón y el colegio americano de cardiólogos (AHA/ACC) el cual busca analizar el riesgo de desarrollar eventos cardiovasculares duros como lo son infarto agudo de miocardio no fatal, muerte de origen coronario e ictus fatal y no fatal (13). Otros modelos buscan identificar la probabilidad de ocurrencia de enfermedad coronaria total por lo cual, además de los eventos anteriormente mencionados, enfermedades como angina inestable también se encuentran dentro de los resultados a predecir $(14,15)$.

- ¿Qué predictores se usarán y cómo se combinarán? Un predictor es un factor de riesgo que mediante su adición al modelo matemático permite predecir el evento cardiovascular, este suele poseer una relación causal con la enfermedad, pero no siempre es así. Estas variables se toman de las características sociodemográficas de la población a estudio, la historia clínica, el examen físico, alteraciones propias de la enfermedad, pruebas aplicables a la misma y tratamiento actual de la condición, estos deben ser definidos claramente antes de la realización de la investigación, se deben usar medidas estandarizadas para su cuantificación, tener en cuenta su disponibilidad en el ambiente sanitario general y haberse demostrado su relevancia clínica con el fin de asegurar su aplicabilidad y estabilidad predictiva $(4,7,16)$.

Los modelos suelen usar factores de riesgo mayores como la edad, historia familiar de enfermedad cardiovascular (ECV), tabaquismo, hipertensión, niveles de lipoproteínas de baja densidad (LDL) entre otros, en los cuales se ha determinado su asociación con eventos cardiovasculares como infarto de miocardio; estos predictores también han recibido la denominación de factores tradicionales. Se han identificado otros predictores no tradicionales o nuevos, aunque su habilidad para predecir correctamente el riesgo cardiovascular aún no se ha dilucidado debido a la falta de evidencia en estudios con 
grandes poblaciones (16-19). Existe controversia sobre si usar la diabetes mellitus (DM) como predictor de riesgo en las escalas o si tomar a los pacientes diabéticos como si tuvieran riesgo mayor por lo cual no sería necesario aplicarlas en ellos (20), más adelante se profundiza este tema.

- ¿En qué grupo poblacional se aplicará el modelo? Los datos para permitir crear el modelo deben ser recolectados de resultados de estudios tipo cohorte prospectivo idealmente, aunque esto no suela cumplirse, pueden ser aleatorizados o no, la elección de la población debe basarse en criterios de inclusión los cuales permiten obtener grupo(s) con características sociodemográficas similares y en los cuales se haya visto una tendencia de igualdad en cuanto a la presentación e influencia de los predictores o factores de riesgo que influyen en la presentación de la enfermedad a predecir. Hay que tener en cuenta que a mayor población de estudio mejor es la probabilidad de que los resultados se reflejen en la población objetivo.

El hecho de tomar datos de estudios poblacionales deja dos consideraciones a tener en cuenta, por una parte las características encontradas en un grupo suelen diferir de las de otros grupos debido a factores como la dieta, el medio ambiente, los estilos de vida, recursos económicos, calidad de los servicios de salud, tratamientos utilizados, determinantes genéticos entre otros, además de encontrar variaciones en cuanto a incidencia de eventos cardiovasculares así como en el comportamiento de los factores de riesgo, por ende una escala desarrollada con población de un país al ser aplicado en otra puede sobreestimar o subestimar la probabilidad de ocurrencia de un evento cardiovascular dejando como resultado un tratamiento preventivo poco específico lo cual se puede solucionar de dos formas, desarrollar más escalas, las cuales sean específicas para cada población a valorar obteniendo con ello un tratamiento óptimo, o aplicar solo los modelos que previamente se hayan validado externamente en la población objetivo $(2,7)$.

Por otra parte, las características encontradas en el grupo de estudio pueden variar con el paso del tiempo debido a mejoras tecnológicas las cuales derivan en nuevos y más eficaces métodos diagnósticos y terapéuticos. Esto, unido a políticas en salud pública que beneficien los cambios de estilo de vida y el manejo de ese mismo grupo, hace que los predictores usados varíen en cuanto a incidencia y peso estadístico al momento de predecir la enfermedad en esa misma población pudiendo afectar la estabilidad predictiva del modelo al ser aplicado en la misma o en una población similar.

En consecuencia, los ajustes y la actualización de los algoritmos, la constante revisión y análisis de los factores de riesgo usados y de las nuevas tecnologías diagnósticas, se hace necesario y lleva a pensar en la búsqueda de otros predictores dando como resultado el aumento actual de las investigaciones de nuevos biomarcadores que permitirán identificar el riesgo cardiovascular. Aunque la revisión de éstos últimos escapa al objetivo de esta revisión, es importante mencionar algunas diferencias entre estos y los predictores tradicionales, destacándose por ejemplo que: mientras que los últimos se piden frecuentemente y están fácilmente disponibles en la práctica clínica los biomarcadores no, la mayoría de los predictores tradicionales son también objetivos terapéuticos modificables mientras que los biomarcadores solo permiten ser medidos y con ello predecir el riesgo, adicionalmente estos últimos son más costosos, poseen mayor probabilidad de causar efectos adversos a los pacientes y no son fácilmente incorporables a las herramientas de evaluación del médico durante su práctica habitual $(5,21)$.

\section{- Tiempo en el cual se desea predecir el evento.}

Es necesario pensar en este punto ya que durante la creación de la metodología del estudio que deriva en el desarrollo del modelo, el tiempo durante el cual se sigue la población determinará el tiempo durante el cual el modelo podrá predecir dichos eventos, adicional a ello se debe pensar en la utilidad clínica del intervalo que se decida usar. En este punto dos variantes han surgido: la de predicción a 5 o 10 años la cual es la más común y a toda la vida, la cual se enfoca en determinar la probabilidad tanto de padecer de una ECV como de morir por ella a lo largo de toda la vida del paciente evaluado (21).

Una vez contestadas las preguntas, el siguiente paso es el de la validación interna, la cual analiza que tan bien se desempeñó el modelo en el grupo de estudio del cual salió, se evalúa su rendimiento y utilidad para realizar el ajuste correspondiente. Ahora bien, cuando esto ocurre en otra población, como se ha mencionado, se denomina validez externa la cual es más problemática que la anterior ya que se debe reclutar una población en la cual se pueda extraer los mismos datos sacados del estudio de desarrollo 
y se debe asumir que los factores de riesgo o predictores que se usan en el modelo y que se extraerán del estudio son iguales en las dos poblaciones.

Para la realización de la validez externa se usan diversas herramientas estadísticas dentro de las cuales se encuentran la discriminación, calibración y re-clasificación. En cuanto al primer ítem, la discriminación, permite clasificar correctamente separando los sujetos en alto y bajo riesgo de desarrollar el evento cardiovascular mediante el uso de la C-estadística, encontrándose como aceptables cifras de 0,7 - 0,8 y excelentes de 0,8 - 0,9. La calibración permite evaluar la concordancia entre el riesgo observado y el predicho cuantificando correctamente el riesgo absoluto. Se estima usando el test de Hosmer-Lemeshow o el área bajo la curva. Cabe resaltar que no son las únicas medidas estadísticas para evaluar estos dos parámetros, se puede encontrar el índice de discriminación y coeficiente de discriminación entre otros. La reclasificación ya se ha explicado a lo largo del artículo, permite realizar cambios a las escalas mediante la adición de marcadores o factores de riesgo nuevos posibilitando así el uso de estos predictores en la población de estudio mejorando su estabilidad predictiva (4-7).

\section{Evaluación de riesgo cardiovascular en pacien- tes con diabetes}

Como factor de riesgo cardiovascular, se ha evidenciado que la DM aumenta dos veces el riesgo de padecer enfermedad coronaria (EC) o eventos cerebrovasculares isquémicos y de dos a cuatro veces la mortalidad por los mismos, diversos factores asociados a la DM aumentan el riesgo. Se ha visto la edad como un factor no modificable y a medida que esta aumenta también lo hace el riesgo cardiovascular, en hombres $>35$ años y mujeres $>$ 45 suben en la escala de bajo a moderado riesgo mientras que hombres $>45$ y mujeres $>54$ cambian de moderado a alto riesgo. El género es otro predictor no modificable, se ha demostrado que las mujeres con DM poseen mayor riesgo de eventos cardiovasculares. En cuanto a la historia familiar de EC se ha visto como su adición a los modelos no mejora la discriminación, pero aun así se considera que poseer parientes de primer grado hombres $<55$ años y mujeres $<65$ años puede ser influyente a la hora de padecer EC en diabéticos. En cuanto al tabaquismo, el ser fumador aumenta 6 veces el riesgo en hombres y dos veces en mujeres si el consumo en promedio es de 20 cigarrillos día, adicional se ha visto como el dejar de fumar disminuye significativamente el riesgo de padecer ECV (Tabla 1). Otras condiciones en el paciente diabético afectan de igual manera la evaluación, el padecer por más de 10 años DM, una tasa de filtración glomerular disminuida, proteinuria y microalbuminuria; en este punto no hay datos sobre reclasificación, discriminación, calibración o costo efecto que permitan adicionar estos últimos tres factores a los modelos actuales aunque su presencia junto a la DM incrementan el riesgo; hipertensión arterial, hiperlipidemia, padecer esteatosis hepática no alcohólica, disfunción eréctil, apnea obstructiva del sueño, hiperglucemia crónica e hipoglicemia severa $(20,22-26)$.

Se han desarrollado tres aproximaciones que deben tenerse en cuenta al momento de evaluar el riesgo en diabéticos. La primera hace referencia a la DM como equivalente de riesgo cardiovascular/enfermedad coronaria, la segunda al uso de modelos unificados para diabéticos y no diabéticos, actualmente se han encontrado 12 modelos desarrollados únicamente para población diabética y 33 para población general en la cual se incluye la DM como factor de riesgo, de estos solo $31 \%$ han sido validados externamente hallándose una discriminación aceptable pero con pobre calibración, adicional a ello, solo se encontró una ecuación que toma en cuenta la aplicación del modelo y su impacto en cuanto a tratamiento y prevención de la ECV. La tercera aproximación hace referencia a un enfoque separado del paciente diabético y no diabético $(4,6,13)$.

Desde el estudio de Haffner et al (27) se considera a la DM como equivalente de riesgo cardiovascular/enfermedad coronaria, lo que significa que un paciente diabético sin ECV tiene un riesgo similar de padecer ECv que un paciente que un paciente sin DM que ya sufrio uno, es decir, que el paciente diabetico en los proximos 10 años tendra un $20 \%$ de riesgo de desarrollar algun ECV, por lo cual diversas guías como el Third Report of the National Cholesterol Education Program's Adult Treatment Panel (ATP-III) (28) usan este concepto derivando en el tratamiento farmacológico intensivo inmediato con estatinas dada su condición de diabéticos. Recientes publicaciones han aportado resultados que no avalan este enfoque. Un meta análisis (8) en el cual se analizaron 13 estudios comparando diabéticos sin infarto previo y pacientes sin DM con infarto previo encontró que los pacientes con DM tenían un $43 \%$ menos de probabilidad de poseer un ECV 
TABLA 1. Factores modificables y no modificables en relación con el riesgo cardiovascular.

\begin{tabular}{|c|c|c|}
\hline \multirow{5}{*}{ Aumenta } & 6 y 2 veces el riesgo respectivamente) & Hombres $>35$ años \\
\hline & \multirow{4}{*}{ Padecer DM durante $>10$ años } & Moderado a alto riesgo \\
\hline & & Mujeres $>54$ años \\
\hline & & Mujeres con DM \\
\hline & & $\begin{array}{l}\text { Familiares de primer grado con DM: } \\
\text { Hombres }<55 \text { años } \\
\text { Mujeres }<65 \text { años }\end{array}$ \\
\hline
\end{tabular}

DM: Diabetes Mellitus

Fuente: construida por los autores a partir de los siguientes articulos: Bertoluci M, Rocha V. Cardiovascular risk assessment in patients with diabetes. Diabetology \& Metabolic Syndrome. 2017 Apr;9(25). Pan A, Wang Y, Talaei M, Hu FB. Relation of smoking with total mortality and cardiovascular events among patients with diabetes mellitus: a meta-analysis and systematic review. Circulation. 2015 Dec;132(19):1795-1804. González-Clemente J, Palma S, Arroyo J, Vilardell C, Caixàs A, Giménez-Palop O et al. ¿La diabetes mellitus es un equivalente de riesgo coronario? Resultados de un metaanálisis de estudios prospectivos. Revista Española de Cardiología. 2007;60(11):1167-76. James PA, Oparil S, Carter BL, Cushman WC, Dennison-Himmelfarb C, Handler J, et al. Evidence-based guideline for the management of high blood pressure in adults: report from the panel members appointed to the Eighth Joint National Committee. JAMA. 2014 Feb; 311 (5): 507-20. Levit R, Wenger N. High Risk, High Stakes: Optimizing Cardiovascular Risk Assessment in Women. Current Cardiovascular Risk Reports. 2012 Apr; 6(2):176-184. Steiropoulos P. Is There Evidence of Early Vascular Disease in Patients with Obstructive Sleep Apnoea Without Known Comorbidities? Preliminary Findings. The Open Cardiovascular Medicine Journal. 2013;6(1):61-68.

en comparación con el grupo de control (OR:0.56, 95\% CI 0.53-0.60), aunque no se pudieron obtener datos específicos para mujeres y hombres, por edad o en población asiática. Estudios posteriores como el de Rana et al (29) en el cual se analizó de manera prospectiva una cohorte de 1.586.061 adultos de edades entre 30-90 años dividida en cuatro grupos, sin DM o EC, con DM pero sin EC, con EC sin DM y con DM y EC halló que los pacientes con DM solo tenían el mismo riesgo que los pacientes con EC después de llevar un tiempo mayor o igual a diez años de duración con la DM, aunque estos tenían el doble de riesgo que pacientes sin DM y sin EC, pese a esto, los autores manifiestan que esos resultados no son suficientes para realizar cambios en cuanto a guías de tratamiento.
Por otra parte, resultados del estudio RUTH (30) realizado específicamente en población femenina encontró que mujeres con DM sin EC poseen menor riesgo de padecer eventos cardiovasculares y coronarios no fatales, pero poseen igual riesgo de padecer eventos cardiovasculares fatales similares a pacientes sin DM con EC previa. Otro meta análisis (23) comparó un grupo de pacientes con DM sin ECV (15000) vs pacientes sin DM con ECV (22000) encontrando que en ambos grupos existe un riesgo aumentado, pero difieren en el sexo, pacientes masculinos con DM sin ECV poseen menor riesgo que el grupo de comparación ( $\mathrm{p}=<0.054)$, en mujeres con DM sin ECV se encontró un riesgo aumentado, aunque no significati- 
vo, esto probablemente debido a la mayor incidencia de factores de riesgo clásicos hallados en este grupo poblacional.

En cuanto al enfoque unificado, se cuenta como si no hubiese interacción entre el estado de la DM y otros factores de riesgo, en otras palabras, si todo lo demás está igual, un paciente diabético frente a un no diabético no siempre tiene un riesgo alto de padecer un evento. Este modelo es en el que actualmente se basan escalas como la de Framingham $(4,14)$. En este punto se puede observar como se ha dejado de adoptar la aproximación de equivalencia la cual lo dejaba como un paciente de alto riesgo y se ha comenzado a estratificar buscando dar un manejo más certero, así diversas guías como la de la AHA/ACC del 2013 (13), la asociación americana de diabetes (ADA) del 2016 (31) y la de la sociedad europea de cardiología (ESC) del mismo año (32) adoptan este enfoque. En la primera, para la evaluación del riesgo cardiovascular (13), se toma a la DM como un factor más para la ecuación y no como un equivalente, se basa en la estimación global del riesgo, pacientes con DM tipo 1 o 2, con edades entre 4075 años con niveles de LDL entre $70-189 \mathrm{mg} / \mathrm{dL}$ se debe dar terapia intensiva si se encuentra alto riesgo $(>7.5 \%)$ y terapia moderada con estatinas si se encuentra en bajo después de usar el modelo predictivo dado por ellos, esto apoyado por su guía para el tratamiento del colesterol del mismo año (33) en donde recomiendan el uso de terapia de moderada intensidad con estatinas para diabéticos entre 40 y 75 años previo análisis de efectos adversos, beneficios del tratamiento, interacciones medicamentosas y preferencias del paciente, para las edades $<40$ años ó $>75$ años proponen dar manejo previa individualización del paciente. Cabe resaltar que la terapia intensiva está definida como aquella que permita disminuir los niveles de $\mathrm{LDL}>50 \%$ mientras que la moderada los disminuye entre un $30-50 \%$.

En la guía dada por la ADA (31) la aproximación está basada en la búsqueda de factores de riesgo y no en el uso de un modelo predictor, se debe analizar en el paciente la edad, antecedentes de ECV y factores de riesgo como niveles de LDL $<100 \mathrm{mg} / \mathrm{dL}$, hipertensión arterial, ser fumador, obesidad o sobrepeso y antecedentes familiares de ECV. Recomienda el manejo mediante terapia intensiva con estatinas en pacientes con ECV previo, sin importar la edad, así como en pacientes sin ECV entre 40-75 años con alguno de los factores de riesgo antes mencionados, en pacientes adultos mayores sin factores de riesgo ni pre- vios ECV dar terapia moderada, y en pacientes adultos jóvenes, sin las variables anteriores, se pueden hacer cambios en el estilo de vida sin dar terapia con estatinas en un inicio. En la guía colombiana (12) se adoptan diversas recomendaciones de la AHA/ACC del 2013 como es la estratificación de los pacientes para dar terapia con estatinas aunque no promulgan el uso del modelo desarrollado por estos debido a la falta de evidencia que demuestre su validez en población de este país, consideran candidatos para manejo con estatinas, a todo paciente diabético, para manejo intensivo aquellos diabéticos $>40$ años con LDL $>70 \mathrm{mg} / \mathrm{dL}$ que adicionalmente posean algún factor de los ya descritos que eleve el riesgo, para manejo moderado aquellos que no cumplan con los anteriores criterios.

En cuanto al enfoque separado se toma en consideración la creación de ecuaciones diferentes para diabéticos y no diabéticos, el ejemplar más significativo de esta aproximación es el derivado del estudio UKPDS (34) el cual busca predecir el riesgo a 10 años, se usaron cohortes de Grecia, Reino Unido, España y China para su desarro1lo, incluye predictores como la edad, duración de la DM, genero, tensión arterial sistólica, colesterol total, niveles de lipoproteínas de alta densidad (HDL), ser fumador, etnicidad y presencia de fibrilación auricular. En un estudio que usó tres cohortes europeas y en el cual se aplicaron 10 modelos para predicción del riesgo entre ellos el UKPDS encontró que todos sobreestiman el riesgo, pero después de realizarse la recalibración todos fueron validados para esta población (35).

Todo lo anterior permite ver que aún no hay un consenso entre si se debe o no clasificar al paciente diabético como riesgo alto, moderado o bajo y por ende brindar un tratamiento precoz en la totalidad de los pacientes con estatinas, cuyos efectos secundarios pueden resultar contraproducentes para el paciente; o reservar dicho manejo para pacientes con alto riesgo cardiovascular, por lo que se insiste en la falta de estudios que apoyen dichas teorías, utilizando como población a pacientes Colombianos $(4,8,10,36)$.

Así, la evidencia demuestra la relación existente entre DM y ECV pero esta difiere ampliamente en cuanto a la aproximación que debe tenerse en estos pacientes, el manejarlos a todos como población de alto riesgo es una práctica que está cambiando debido a la diferente evidencia que ha surgido. En caso de estratificación ésta se puede realizar usando solamente los factores de ries- 
go o mediante el uso de modelos predictivos. Aún hace falta indagar sobre cómo afectan los factores de riesgo emergentes, el cómo se pueden aprovechar condiciones como la tasa de filtración glomerular, la proteinuria y la albuminuria, así como el rol de los nuevos biomarcadores y técnicas usadas para la detección de aterosclerosis en estos pacientes $(20,37,38)$.

\section{Modelos de riesgo existente para población general}

Para escoger el modelo adecuado que permita identificar de mejor manera el riesgo en un paciente, el médico debe tener diversas variables en cuenta, dentro de estas, escoger el modelo que derive de la población a la cual se aplicará o haber sido ajustado a esta, el que obtenga como resultado el riesgo absoluto; y aquí cabe recalcar que el resultado en porcentaje arrojado por las escalas no se debe interpretar asumiendo que si un modelo arroja un resultado de $7 \%$ significa que el riesgo de tener un evento cardiovascular es del $7 \%$ en ese paciente, la manera correcta de interpretarlo es que sobre un total de 100 personas similares a él, 7 padecerán dicho un evento y 93 no $(4,5)$. El modelo debe proveer, además, representaciones gráficas a los pacientes y se debe tener en cuenta la presentación del mismo hacia el personal de salud ya que debe ser fácilmente usable, se prefieren las aplicaciones para celulares o calculadoras en páginas web, también debe ser entendible, por lo cual, debe esconder los detalles matemáticos y explicar qué herramientas metodológicas se usaron para predecirlo, en lo posible debe estimar el beneficio de la intervención a realizar $(16,39,40)$.

Lo anterior es especialmente importante ya que, como fue demostrado en una revisión sistemática (41), la diversidad de modelos existentes es enorme, 363 herramientas diferentes para la estratificación cardiovascular fueron halladas de las cuales solo 132 han sido validadas externamente, se encontró que la mayoría de estos modelos fueron diseñados en Europa, los predictores más comunes fueron la edad y ser fumador y la mayoría de estos presentaban predicciones a 10 años. El problema principal encontrado fue el exceso de modelos existentes y el no soporte basado en la evidencia de la mayoría de ellos debido a varios factores, a saber: la no presentación correcta de los mismos debida a la pérdida de los datos o la no publicación de la metodología usada en los estudios de desarrollo, así como, la falta de estudios que demuestran el impacto del uso de estos en la población general. Adi- cional a esto, de los 132 estudios sobre validación externa la discriminación y calibración sólo fueron reportadas en un $65 \%$ y $58 \%$ respectivamente. Los autores reportan que tres años después de la recolección de datos al momento de la publicación se encontraron más de 4000 artículos nuevos los cuales cumplen con los parámetros de búsqueda y análisis lo cual lleva a pensar que la cantidad de modelos desarrollados sigue en aumento.

Debido a esta gran cantidad de herramientas se puede hacer un acercamiento, sobre cuál de ellos usar analizando lo dicho por las diferentes guías existentes, una revisión sistemática de estas, realizada en el 2016, analizó las que cumplían con el rigor metodológico de desarrollo necesario, encontrándose 17 guías sobre la evaluación de riesgo cardiovascular de las cuales 7 tratan sobre disglucemia, 2 sobre dislipidemia, 3 sobre hipertensión y 5 hacen referencia al tamizaje del riesgo cardiovascular fatal. La mayoría de estas recomiendan el uso de modelos de riesgo para guiar el manejo farmacológico, estos deben integrar predictores como la edad, el sexo, ser fumador, la tensión arterial y el nivel de lípidos, así como la necesidad de incorporar uno nuevo, la etnicidad, pero no hay consenso sobre cuál de todos los modelos existentes escoger. Las 5 guías sobre tamizaje de riesgo cardiovascular promueven el uso de las siguientes escalas: QRISK2, SCORE (systematic coronary risk evaluation), Framingham a 5 o 10 años, la ecuación desarrollada por la AHA/ACC, y el score de riesgo de Reynolds. Tampoco demuestran consenso en cuanto al tiempo al cual deben predecir los modelos, si a 5, 10 años o a toda la vida, cada cuánto revalorar el riesgo en un paciente o los puntos de corte y algoritmos a usar para implementar el manejo con estatinas (Tabla 2) (42).

Aunque la herramienta más usada en Latinoamérica es el Framingham, dos modelos han sido construidos usando esta población, uno con pacientes chilenos en el 2009 el cual estima el riesgo a 10 años y otro en Puerto Rico el cual lo estima a 8 años. Ambos usan factores de riesgo tradicionales y no tradicionales, estiman el riesgo relativo, pero solo el primero estima el riesgo absoluto (2). En el estudio CARMELA (43), auspiciado por Pfizer, en el cual se busca caracterizar a la población latinoamericana se aplicó el Framingham para tipificar a la población de 7 ciudades de esta región, aunque los autores reconocen que el modelo no se ha validado, se encontró una distribución heterogénea del riesgo cardiovascular, así como, de la prevalencia de los factores de riesgo usando la evaluación dada por el ATP III. La mayor prevalencia de DM se en- 
TABLA2. Escalas usadas por cinco guías sobre tamizaje de riesgo cardiovascular junto con los predictores de base utilizados en los modelos de riesgo.

\begin{tabular}{lc}
\hline Escalas & Predictores \\
\hline QRISK & Edad \\
\hline SCORE & Sexo \\
\hline Framingham 5 ó 10 años & Tabaquismo \\
AHA/ACC & Tensión arterial \\
\hline Score de riesgo de Reynolds & Cifras de lípidos \\
\hline
\end{tabular}

SCORE: Systematic Coronary Risk Evaluation; AHA/ACC: Asociación Americana del Corazón/ Colegio americano de Cardiología

Fuente: Construida por los autores en base a: Khanji M, Bicalho V, van Waardhuizen C, Ferket B, Petersen S, Hunink M. Cardiovascular Risk Assessment. Annals of Internal Medicine. 2016;165(10):713.

contró en Ciudad de México y Bogotá, donde se encuentran la hipertensión e hipercolesterolemia como unos de los factores que más influyen en el riesgo de padecer un evento cardiovascular mayor, aunque la combinación de hipertensión y DM demostró que la aumenta aún más. Un meta análisis (44) con 13142 personas demostró cómo el modelo de Framingham sobreestima el riesgo en Latinoamérica incluyendo el caribe y población hispana residente en Estados Unidos, este estudio además evidenció las pocas investigaciones referentes a validación aplicadas sobre esta población, solo 5 cumplían con una adecuada metodología aunque no se buscaron estudios no indexados, además de encontrar una amplia heterogeneidad entre la misma población probablemente debido a la dieta, estilos de vida e incluso genética y ascendencia todo lo cual limitaría el resultado de cada estudio a la población en la cual fue ajustada, si se quisiera aplicar esa escala ya calibrada para una población de Latinoamérica a otra en la cual no se haya realizada se deben hacer los ajustes correspondientes $(2,44)$.

\section{Los modelos de riesgo en Colombia}

En Colombia las escalas más usadas son las de Framingham, PROCAM, SCORE y AHA/ACC $(9,45)$ y la recomendada por la guía del ministerio de salud es la de Framingham modificada (12) las cuales serán ampliadas a continuación.

El predictor Framingham para riesgo cardiovascular, aplicado en pacientes blancos estadounidenses que asistieron al ciclo de examen $11^{\circ}$ de la primera cohorte origi- nal (1968-1971) o la tercera de (1984-1987) y no habían presentado una ECV de 30-74 años, es usado para estimar a 10 años la presencia de una cardiopatía coronaria absoluta; utilizando los siguientes predictores: Antropometría, presión arterial, colesterol total, HDL en suero, tabaquismo, DM o uso de insulina o medicamentos hipoglucemiantes orales, uso de medicamentos antihipertensivos. La información de eventos cardiovasculares se determinó a partir de las historias clínicas, exámenes físicos en la clínica del estudio, registro de hospitalizaciones y los siguientes puntos de corte: bajo riesgo: $0-6 \%$ mediano riesgo:6-20\% y alto riesgo $>20 \%$. En ésta población la escala demostró una muy buena calibración y discriminación, a futuro busca adicionar ciertos factores de riesgo que puedan ayudar a mejorar aún más su discriminación y calibración (14).

Por otra parte, PROCAM, fue aplicada a hombres de procedencia alemana entre 35-65 años en los años comprendidos entre 1979-1985 sin antecedentes de infarto agudo miocardio, evento cerebrovascular, cardiopatía isquémica o angina de pecho, con el fin de estimar eventos coronarios mayores (muerte cardiaca repentina, o un infarto de miocardio mortal o no mortal evidenciado en ECG o enzimas cardiacas) a 10 años, utilizando como predictores la edad, colesterol LDL, tabaquismo, colesterol HDL, presión arterial sistólica, antecedentes familiares de infarto del miocardio prematuro, DM y triglicéridos demostrando adecuada validación interna. Los puntos de corte fueron $<20 \%$ bajo riesgo, $>=20 \%$ alto riesgo (46). 
Las tablas para riesgo cardiovascular SCORE-Deutschland actualizadas fueron aplicadas a una población alemana entre los años 1998-2002, para estimar el riesgo existente de enfermedad cardiovascular fatal a diez años. Para ello utilizó como predictores: sexo, edad, presión arterial sistólica, tabaquismo y colesterol total, teniendo como puntos de corte: SCORE -bajo: $<1 \%$, SCORE- medio: $>=1<5 \%$, SCORE alto: $>=5,<10 \%$ y SCORE- muy alto: $>=10 \%$. La última actualización que se había realizado de ésta fue en el año 2005, no obstante, dada la mejoría en la calidad de vida y reducción en la tasa de mortalidad secundaria a eventos cardiovasculares fatales por parte de la población alemana, se sugirió una nueva calibración para evitar una sobreestimación del riesgo cardiovascular, la cual evidenció permitir una mayor exactitud en la estimación del mismo $(8,47)$.

Finalmente en el 2013 se postuló una ecuación de la AHA, de la cual ya se ha hablado, actualizada para estimar por sexo y raza específicos el riesgo de desarrollar un primer evento cardiovascular aterosclerótico a diez años, utilizando como población a pacientes hombres y mujeres afroamericanos y blancos no hispanos de 40-79 años de edad; teniendo como variables: raza, sexo, edad, colesterol total, colesterol de lipoproteínas de alta densidad, presión arterial sistólica, DM y estado actual de tabaquismo. El punto de corte para riesgo cardiovascular alto fue $>=$ $7.5 \%$. Finalmente, concluyeron que éstas ecuaciones específicas para raza-sexo con un grado de recomendación B por parte de NHLBI en cuanto a predecir el riesgo a 10 años de un primer evento ASCVD podía ser utilizado en los blancos y afroamericanos no hispanos, de 40 a 79 años de edad y con una recomendación de la NHLBI grado E se sugirió el uso de las ecuaciones específicas del sexo pueden ser consideradas para la estimación del riesgo en pacientes de poblaciones distintas a los afroamericanos y blancos no hispanos (13). Se ha demostrado como la escala de riesgo de la ACC/AHA en población multirracial constituida por blancos, negros, chinos e hispanoamericanos, tanto en hombres como en mujeres de los cuatro grupos sobreestimó el riesgo de ECV de igual manera esto se ha visto en población estadounidense debido principalmente a la población que se usó para su desarrollo ya que está derivada de 5 cohortes mayoritariamente entre los años 1960 - 1980 por lo cual cambios probables ya explicados tanto en la incidencia como en el peso estadístico de los predictores usados pueden ser los causantes de la sobreestimación (21,49-51).
En Colombia, un único estudio de validación (11) de cohorte histórica evaluó 1013 pacientes de entre 30-74 años usando los modelos de Framingham y PROCAM encontrándose que el primer modelo presenta mala calibración y discriminación sobreestimando el riesgo, mientras que la ecuación de PROCAM ajustada por sexo presenta una mejor capacidad de discriminación $(0,74)$, con buena calibración. El estudio cuenta con diversas limitaciones dentro de las cuales destaca la falta de pacientes de alto riesgo por lo cual no se pueden extrapolar los resultados a ésta población, adicional a ello la muestra fue extraída de una clínica de prevención y atención primaria la cual posee en su mayoría población militar o exmilitar y sus beneficiarios, por lo cual, el comportamiento de los predictores puede ser diferente del de la población general colombiana, de hecho se encontró menor incidencia de DM que la evidenciada en el entorno colombiano e incluso que la mostrada por el estudio CARMELA así como menor incidencia de tabaquismo dentro de la población de estudio. Adicional a ello, el ser una muestra histórica también puede llevar a pensar que el comportamiento de los predictores puede haber cambiado comparado con el que puede encontrarse en la población actual.

Aunque los autores del estudio anterior recomiendan el uso de la ecuación de PROCAM sobre la de Framingham dada la validez externa alcanzada por ésta, la guía para el tratamiento de las dislipidemias comisionada por el Ministerio de Protección social y Colciencias (12) basándose únicamente en los resultados del estudio anterior, recomienda el uso del modelo Framingham ajustado para Colombia, el cual consiste en la multiplicación del resultado por 0,75 , sobre PROCAM debido a que su uso está más extendido, ha sido propuesta por dos consensos de la Sociedad Colombiana de Cardiología y existe mayor familiaridad del cuerpo de salud ante esta que con el modelo PROCAM. En un estudio posterior (45) se comparó el uso de los modelos SCORE, ACC/AHA 2013, y Framingham modificado para Colombia (por 0,75 ) encontrando que los primeros modelos no estiman correctamente el riesgo, aunque tomando como punto de comparación, la ecuación de la AHA/ACC posee mejor concordancia con la recomendada por la guía para Colombia que el SCORE, aunque desestima el riesgo predicho.

Todo lo anterior deja cierta incertidumbre a la hora de aplicar dichas herramientas en población colombiana. Es claro que el médico debe escoger modelos que estén va- 
lidados en esta población, requiere que la explicación en cuanto a metodología, calibración y discriminación tanto de los estudios de desarrollo del modelo como los aplicados a pacientes colombianos se encuentren publicados, sean válidos y las herramientas sean amigables tanto con el usuario como con el paciente. Considerando esto, se recomiendan nuevos estudios sobre el tema y el uso solo de modelos validados previamente en Colombia para alcanzar adecuadamente las recomendaciones dadas por la OMS disminuyendo tanto la incidencia de ECV, mejorando la calidad de vida de los pacientes y logrando disminución en los costos en el sistema de salud colombiano actual.

\section{Referencias}

1. M Moons, K G. Risk prediction models: I. Development, internal validation, and assessing the incremental value of a new (bio) marker. Heart. 2012;98(9):683-690.

2. Zhao D, Liu J, Xie W, Qi Y. Cardiovascular risk assessment: a global perspective. Nature Reviews Cardiology. 2015;12(5):301311.

3. Otgontuya D, Oum S, Buckley B, Bonita R. Assessment of total cardiovascular risk using WHO/ISH risk prediction charts in three low and middle income countries in Asia. BMC Public Health. 2013;13(1):539-551.

4. Echouffo-Tcheugui, Justin B. et al. On the importance of global cardiovascular risk assessment in people with type 2 diabetes. PCDE. 2013;7(2):95-102

5. Lloyd-Jones, DM. Cardiovascular Risk Prediction: basic concepts, current status, and future directions. Circulation. 2010; 121(15):1768-1777.

6. Petretta M, Cuocolo A. Prediction models for risk classification in cardiovascular disease. European Journal of Nuclear Medicine and Molecular Imaging. 2012;39(12):1959-1969.

7. Prieto-Merino D, Pocock S. The science of risk models. European Journal of Preventive Cardiology. 2012;19(2):7-13.

8. Van Dieren. et al. Prediction models for the risk of cardiovascular disease in patients with type 2 diabetes: a systematic review. Heart. 2012;98(5):360-369.

9. Martín-Timón I. Type 2 diabetes and cardiovascular disease: Have all risk factors the same strength?. World Journal of Diabetes. 2014;5(4):444-70.

10. Bulugahapitiya U, Siyambalapitiya S, Sithole J, Idris I. Is diabetes a coronary risk equivalent? :Systematic review and metaanalysis. Diabetic Medicine. 2009;26(2):142-148.

11. Muñoz O, Rodríguez N, Ruiz A, Rondon M. Validación de los modelos de predicción de Framingham y PROCAM como estimadores del riesgo cardiovascular en una población colombiana. Revista Colombiana de Cardiología. 2014;21(4):202-212.
12. Muñoz $\mathrm{O}$, García $\mathrm{A}$, Fernández $\mathrm{D}$ et al. Guía de práctica clínica para la prevención, detección temprana, diagnóstico, tratamiento y seguimiento de las dislipidemias: evaluación del riesgo cardiovascular. Revista Colombiana de Cardiología. 2015;22(6):263-269.

13. Goff D, Lloyd-Jones D, Bennett G, Coady S, D'Agostino R, Gibbons R et al. 2013 ACC/AHA Guideline on the Assessment of Cardiovascular Risk . Circulation. 2013;63(25): 2935-2959.

14. D'Agostino R, Vasan R, Pencina M, Wolf P, Cobain M, Massaro J et al. General Cardiovascular Risk Profile for Use in Primary Care: The Framingham Heart Study. Circulation. 2008 Feb;117(6):743753.

15. Múñoz O, García Á, Fernández D, Higuera A, Ruiz Á, Aschner P et al. Clinical practice guidelines for prevention, early detection, diagnose, treatment and follow-up of dyslipidemias: farmacological treatment with statins. Revista Colombiana de Cardiología 2015;22(1):14-21.

16. Carbone R, Algahim M, Rizzo S, Monselise A, Dart A, Almassi G, Gutterman D. Cardiovascular risk in patients without known cardiovascular disease. Tumori Journal. 2014;18(3):365-373.

17. Yusuf S, Hawken S, Ônpuu S, Dans T, Avezum A, Lanas F et al. Effect of potentially modifiable risk factors associated with myocardial infarction in 52 countries (the INTERHEART study): case-control study. Lancet. 2017;364(9438):937-952

18. Alagona P,Ahmad T. Cardiovascular Disease Risk Assessment and Prevention. Medical Clinics of North America. 2015;99(4):711731.

19. Mortensen MB, Afzal S, Nordestgaard BG, et al. Primary prevention with statins: ACC/AHA risk-based approach versus trial-based approaches to guide statin therapy. JACC. 2015;66 (24):2699-2709.

20. Bertoluci M, Rocha V. Cardiovascular risk assessment in patients with diabetes. Diabetology \& Metabolic Syndrome. 2017;9(25):113.

21. Pletcher M, Moran A. Cardiovascular Risk Assessment. Med Clin N Am. 2017 Jul; 101 (4): 673-88

22. Pan A, Wang Y, Talaei M, Hu FB. Relation of smoking with total mortality and cardiovascular events among patients with diabetes mellitus: a meta-analysis and systematic review. Circulation. 2015;132(19):1795-1804.

23. González-Clemente J, Palma S, Arroyo J, Vilardell C, Caixàs A, Giménez-Palop $\mathrm{O}$ et al. ¿La diabetes mellitus es un equivalente de riesgo coronario? Resultados de un metaanálisis de estudios prospectivos. Revista Española de Cardiología. 2007;60(11):11671176 .

24. James PA, Oparil S, Carter BL, Cushman WC, Dennison-Himmelfarb C, Handler J, et al. Evidence-based guideline for the management of high blood pressure in adults: report from the panel members appointed to the Eighth Joint National Committee. JAMA. 2014;311(5): 507-520.

25. Levit R, Wenger N. High Risk, High Stakes: Optimizing Cardiovascular Risk Assessment in Women. Current Cardiovascular Risk Reports. 2012;6(2):176-184. 
26. Steiropoulos P. Is There Evidence of Early Vascular Disease in Patients with Obstructive Sleep Apnoea Without Known Comorbidities? Preliminary Findings. The Open Cardiovascular Medicine Journal. 2013;6(1):61-68.

27. Haffner S, Lehto S, Ronnemaa $\mathrm{T}$ et al. Mortality from coronary heart disease in subjects with type 2 diabetes and in nondiabetic subjects with and without prior myocardial infarction. NEJM. 1998;339 (1998) 229-234.

28. Third Report of the National Cholesterol Education Program (NCEP) Expert Panel on Detection, Evaluation, and Treatment of High Blood Cholesterol in Adults (Adult Treatment Panel III) Final Report. Circulation. 2002;106(25):3143-3421.

29. Rana et al. Diabetes and Prior Coronary Heart Disease are Not Necessarily Risk Equivalent for Future Coronary Heart Disease Events. JGIM. 2016;31(4):387-393.

30. Daniels et al. Is Diabetes Mellitus a Heart Disease Equivalent in Women? Results From an International Study of Postmenopausal Women in the Raloxifene Use for the Heart (RUTH) Trial Circulation: Cardiovascular Quality and Outcomes. Circulation. 2013;6(2):164-170.

31. Standards of Medical Care. Diabetes-2016: summary of revisions. Diabetes Care. 2016;39(1):1-2.

32. Piepoli MF, Hoes AW, Agewall S, Albus C, Brotons C, Catapano AL, et al. 2016 European guidelines on cardiovascular disease prevention in clinical practice: the sixth joint task force of the European Society of Cardiology and Other Societies on Cardiovascular Disease Prevention in Clinical Practice (constituted by representatives of 10 societies and by invited experts)Developed with the special contribution of the European Association for Cardiovascular Prevention \& Rehabilitation (EACPR). Eur Heart J. 2016;37(29):2315-2381.

33. Stone N, Robinson J, Lichtenstein A, Bairey C et al. 2013 ACC/ AHA Guideline on the Treatment of Blood Cholesterol to Reduce Atherosclerotic Cardiovascular Risk in Adults. JACC. 2014;63(25):2889-2934.

34. Kothari V, Stevens R, Adler A, et al. UKPDS 60 risk of stroke in type 2 diabetes estimated by the UK prospective diabetes study risk engine, Stroke. 2002;33(7):1776-1781.

35. Van der Leeuw J, Van Dieren S, Beulens J, Boeing H, Spijkerman A, Van der Graaf $Y$ et al. The validation of cardiovascular risk scores for patients with type 2 diabetes mellitus. Heart. 2014;101(3):222-229.

36. Paynter N. Cardiovascular Risk Prediction in Diabetic Men and Women Using Hemoglobin A1c vs Diabetes as a High-Risk Equivalent. Archives of Internal Medicine. 2011;171(19):1712-18.

37. Allan GM, Nouri F, Korownyk C, Kolber MR, Vandermeer B, McCormack J. Agreement among cardiovascular disease risk calculators. Circulation. 2013;127(19):1948-1956.
38. Helfand M, Buckley DI, Freeman M, et al. Emerging risk factors for coronary heart disease: a summary of systematic reviews conducted for the U.S. Preventive Services Task Force. Ann Intern Med. 2009;151(7):496-507.

39. Allan G, Garrison S, McCormack J. Comparison of cardiovascular disease risk calculators. Current Opinion in Lipidology. 2014;25(4):254-265.

40. Graham I, D'Agostino R. Therapeutic strategies in cardiovascular risk. Glob. Heart. 2013;8(1):11-23.

41. Damen J, Hooft L, Schuit E, Debray T, Collins G, Tzoulaki I et al. Prediction models for cardiovascular disease risk in the general population: systematic review. BMJ. 2016;16;353(2416):1-11.

42. Khanji M, Bicalho V, van Waardhuizen C, Ferket B, Petersen S, Hunink M. Cardiovascular Risk Assessment. Annals of Internal Medicine. 2016;165(10):713.

43. Schargrodsky et al. The CARMELA Study. The American Journal of Medicine. 2008;121:58-65.

44. Cortes-Bergoderi M, Thomas R, Albuquerque F, Batsis J, Burdiat $\mathrm{G}$, Perez-Terzic $\mathrm{C}$ et al. Validity of cardiovascular risk prediction models in Latin America and among Hispanics in the United States of America: a systematic review. Revista Panamericana de Salud Pública. 2012;32(2):131-139.

45. Muñoz O, Ruiz J, Mariño A. Bustos M. Concordancia entre los modelos de SCORE y Framingham y las ecuaciones AHA/ACC como evaluadores de riesgo cardiovascular. Revista Colombiana de Cardiología. 2016;24(2): 110-116.

46. Assmann G. Simple Scoring Scheme for Calculating the Risk of Acute Coronary Events Based on the 10-Year Follow-Up of the Prospective Cardiovascular Munster (PROCAM) Study. Circulation. 2002;105(3):310-315.

47. Rücker V, Keil U, Fitzgerald A, Malzahn U, Prugger C, Ertl G et al. Predicting 10-Year Risk of Fatal Cardiovascular Disease in Germany: An Update Based on the SCORE-Deutschland Risk Charts. PLoS One. 2016;11(19): e0162188.

48. Merry, A. H. et al. Risk prediction of incident coronary heart disease in The Netherlands:re-estimation and improvement of the SCORE risk function. SAGE Journals.2012;19(4), 840-848.

49. DeFilippis A, Young R, McEvoy J, Michos E, Sandfort V et al. Risk score overestimation: the impact of individual cardiovascular risk factors and preventive therapies on the performance of the American Heart Association-American College of Cardiology-Atherosclerotic Cardiovascular Disease risk score in a modern multi-ethnic cohort. European Heart Journal.2017;38(8):598-608.

50. Cook N, Ridker P. Calibration of the Pooled Cohort Equations for Atherosclerotic Cardiovascular Disease. Annals of Internal Medicine. 2016;165(11):786-794.

51. Muntner P, Colantonio L, Cushman M et al. Validation of the atherosclerotic cardiovascular disease pooled cohort risk equations. JAMA. 2014;311(14):1406-1415. 